\title{
An explicit method for modeling lossy and dispersive transmission lines
}

\section{P. Palà-Schönwälder and J.M. Miró-Sans}

UPC - Department of Signal Theory and Communications

P.O. Box 30.00208080 Barcelona Spain

\section{Abstract}

In this paper, an explicit -non iterative- method for modeling lossy and dispersive transmission lines, allowing the inclusion of skin-effect parameters is described. This method, based on multipoint Padé approximation, allows direct implementation to obtain models for existing simulation program -such as SPICEwithout the need of making use of optimization algorithms at any stage. Examples are given to show that the described procedure yields the same accuracy as other existing techniques that do require this iterative approach.

\section{Introduction}

RLCG transmission lines used for circuit interconnection can be characterized as a two-port, by the equations

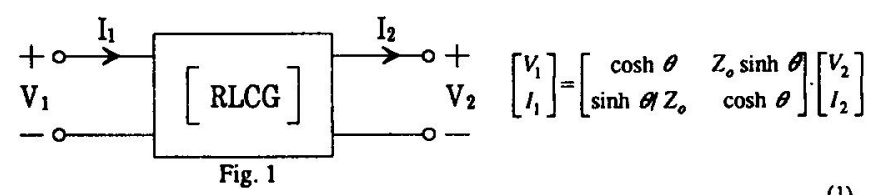

where $\left(V_{1}, I_{1}\right)$ and $\left(V_{2}, I_{2}\right)$ are the voltage and current at the input port -near end-and the output port -far end. The characteristic impedance $\mathrm{ZO}$ and the propagation constant $\theta$ are obtained from the parameters $\mathrm{R}, \mathrm{L}, \mathrm{C}, \mathrm{G}$ and physical line length $\mathrm{l}$ using the expressions

$$
Z_{o}(s)=\sqrt{\frac{R+L s}{G+C s}} \text { and } \theta(s)=l \cdot \sqrt{(R+L s)(G+C s)}
$$

Equation (1) may be rewritten and modeled [1] as
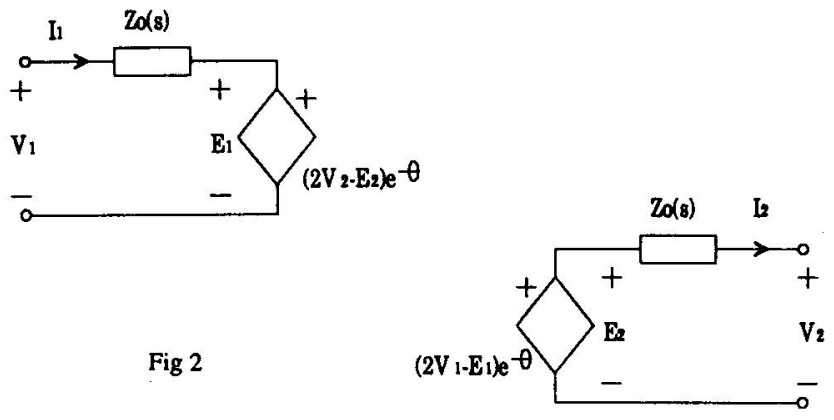

This model may be expanded (figure 3 ) to obtain a circuit that allows direct implementation on standard circuit simulation programs, such as SPICE.

Several techniques for the synthesis of $Z_{0}(s)$ and $F(s)=\exp (-\theta)$ have been described. An explicit method for the synthesis of the characteristic impedance, based on the Padé approximation of $Z_{\delta}(s)$, is described in [1]. Depending on the capacitive or inductive behavior of $Z_{\alpha}(s)$, a rational approximation that is exact about $s \rightarrow \infty$ or $s=0$, respectively, is obtained. The main disadvantage of this technique is that errors are unnecessarily high at low or high frequencies, respectively, requiring more lumped elements for a prescribed accuracy. On the other hand, the method is not suitable for the inclusion of frequencydependent parameters.

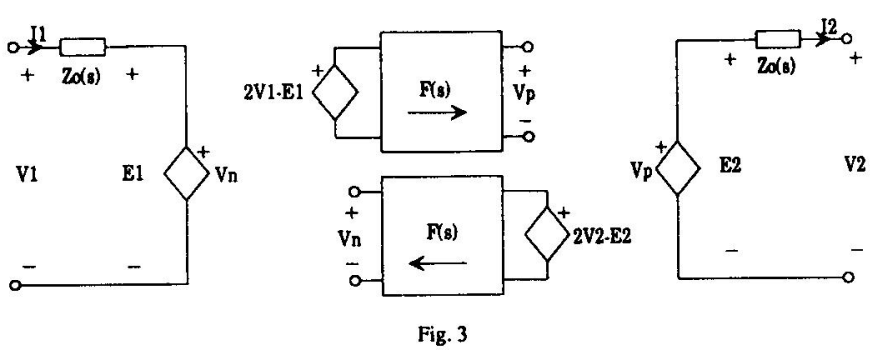

It is possible to overcome these drawbacks making use of nonexplicit methods -those that require iterative minimization algorithms [2],[3]. As a first step before modeling, a rational function that approximates the objective function is computed by an iterative optimization procedure. The corresponding equivalent circuit is then to be synthesized using conventional techniques.

The inclusion of an ideal transmission line (figure 4) to model the major part of the physical delay [2] decreases dramatically the number of lumped elements otherwise needed to model the propagation function $F(s)$.

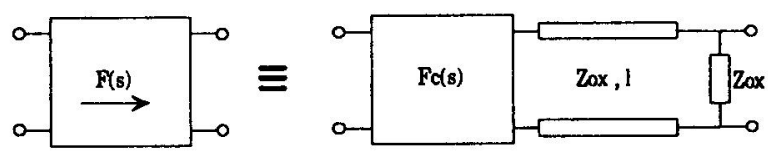

Fig. 4

In this paper, an explicit method for the synthesis of $Z_{0}(s)$ and $F_{C}(s)$, based on the multipoint Pade approximation, is presented. Besides allowing easy implementation, the method described makes it possible to overcome the difficulties inherent to optimization algorithms, such as initial parameter estimation or convergence to local minima, while achieving the same accuracy as obtained with minimization techniques.

\section{Multipoint Padé Approximants}

Problem formulation: Given a function $f(z)$, represented by the $N_{1}, N_{2}, \ldots, N_{R}$ first terms of its corresponding power series expansion about the points $w_{1}, w_{2} \ldots, w_{R}$

$$
\left\{\begin{array}{l}
f(z)=d_{10}+d_{11}\left(z-w_{1}\right)+d_{12}\left(z-w_{1}\right)^{2}+\cdots+d_{1 N_{1}}\left(z-w_{1}\right)^{N_{1}} \\
f(z)=d_{20}+d_{21}\left(z-w_{2}\right)+d_{22}\left(z-w_{2}\right)^{2}+\cdots+d_{2 N_{2}}\left(z-w_{2}\right)^{N_{2}} \\
\quad \vdots \\
f(z)=d_{R 0}+d_{R 1}\left(z-w_{R}\right)+d_{R 2}\left(z-w_{R}\right)^{2}+\cdots+d_{R N_{R}}\left(z-w_{R}\right)^{N_{R}}
\end{array}\right.
$$


find a rational function $H_{m n}(z)$

$$
H_{m, n}(z)=\frac{b_{0}+b_{1} z+b_{2} z^{2}+\cdots+b_{m} z^{m}}{a_{0}+a_{1} z+a_{2} z^{2}+\cdots+a_{n} z^{n}}
$$

such that the $N_{l}, N_{2}, \ldots, N_{R}$ first terms of its power series expansion about the points $w_{1}, w_{3} \ldots, w_{R}$ agree with those of the original function.

It should be noted that, as the number of undetermined coefficients in the rational function is $N=m+n+1$, in general, only $N$ data coefficients may be adjusted.

An efficient method for solving this problem [4] requires the following definitions.

Divided differences are defined as

$$
\begin{aligned}
& f\left[z_{0}\right]=f\left(z_{0}\right), \quad f\left[z_{0}, z_{1}\right]=\frac{f\left(z_{0}\right)-f\left(z_{1}\right)}{z_{0}-z_{1}}, \cdots, f\left[z_{0}, z_{1}, \cdots, z_{r+1}\right]= \\
& \frac{f\left[z_{0}, z_{1}, \cdots, z_{r-1}, z_{r}\right]-f\left[z_{0}, z_{1}, \cdots, z_{r-1}, z_{r+1}\right]}{z_{r}-z_{r+1}}, r=1,2, \cdots
\end{aligned}
$$

If $z_{0}=z_{l}=\ldots=z_{n}$, it is natural to define

$$
\begin{array}{r}
f\left[z_{0}, z_{0}, \cdots, z_{0}\right]=\frac{f^{(r)}\left(z_{0}\right)}{r !} \\
f_{i j}=\left\{\begin{array}{cc}
0, & i>j \\
f\left[z_{i}, \cdots, z_{j}\right], & i \leq j
\end{array}\right.
\end{array}
$$

To simplify notation, let's define $\mathbf{f}_{\mathrm{i}}$ Points $\mathrm{z}_{\mathrm{i}}$ are defined as:

$z_{L(i-1)}=z_{L(i-1)+1}=\cdots=z_{L(i)-1}=w_{i}, \quad 1 \leq i \leq R$, where $L(0)=0, L(i)=\sum_{k=1}^{i}\left(N_{k}+1\right)$

With this definition, the desired approximant, expressed in the form

$$
H_{m, n}(z)=\frac{b_{00}+b_{01}\left(z-z_{0}\right)+\cdots+b_{0 m} \prod_{k=0}^{m-1}\left(z-z_{k}\right)}{a_{00}+a_{01}\left(z-z_{0}\right)+\cdots+a_{0 n} \prod_{k=0}^{n-1}\left(z-z_{k}\right)}
$$

may be obtained from the expressions

$$
\left[\begin{array}{cccc}
f_{0, m+1} & f_{1, m+1} & \cdots & f_{n, m+1} \\
f_{0, m+2} & f_{1, m+2} & \cdots & f_{n, m+2} \\
\vdots & \vdots & \ddots & \vdots \\
f_{0, m+n} & f_{1, m+n} & \cdots & f_{n, m+n}
\end{array}\right]\left[\begin{array}{c}
a_{00} \\
a_{01} \\
\vdots \\
a_{0 n}
\end{array}\right]=\left[\begin{array}{c}
0 \\
0 \\
\vdots \\
0
\end{array}\right],\left[\begin{array}{c}
b_{00} \\
b_{01} \\
\vdots \\
b_{0 m}
\end{array}\right]=\left[\begin{array}{cccc}
f_{0,0} & 0 & \cdots & 0 \\
f_{0,1} & f_{1,1} & \cdots & 0 \\
\vdots & \vdots & \ddots & \vdots \\
f_{0, m} & f_{1, m} & \cdots & f_{m, m}
\end{array}\right]\left[\begin{array}{c}
a_{00} \\
a_{01} \\
\vdots \\
a_{0 n}
\end{array}\right]
$$

In the above system of $n$ linear equations, usually one degree of freedom is available, and it is possible to give an arbitrary value to one of the unknowns. See [5] for an exhaustive treatment of this subject.

Since $m$ and $n$ are usually small (less than 10 ), the procedure of finding $H_{m, n}(z)$ is computationally not expensive.

\section{Synthesis of $Z_{0}(S)$ and $F_{C}(S)$}

From the frequency response plot of the characteristic impedance of a RLCG transmission line, clearly the rational approximation to be found should have the same number of poles and zeros, i.e., $m=n$. Since the objective is to approximate $Z_{\alpha}(s)$ over the whole frequency axis, it is convenient to use a bilinear transformation $s=s_{0} \frac{1-\mathrm{z}}{1+\mathrm{z}}$ or, equivalently $z=\frac{s_{0}-s}{s_{0}+s}$.

This transformation maps the frequency axis on the unit circle, thus enabling to set restrictions for $f \rightarrow \infty$, as this point is mapped onto $z=-1$. Since $Z_{0}(s)$ is symmetric about $s_{0}=(R G / L C)^{1 / 2}$, that is

$$
\frac{Z_{o}\left(s_{o}\right)}{Z_{o}\left(\lambda \cdot s_{0}\right)}=\frac{Z_{o}\left(s_{o} / \lambda\right)}{Z_{o}\left(s_{o}\right)}
$$

for any scalar $\lambda$, it is convenient to use $s_{0}=(R G / L C)^{1 / 2}$ in the bilinear transformation. As a function of $z, Z_{d}(s)$ may be expressed as

$$
\begin{gathered}
\left.Z_{o}(z)=H_{o} \cdot \sqrt{\frac{1+a \cdot z}{1-a \cdot z}}, \text { where } H_{o}=\left(\frac{R}{G}\right)^{1 / 2}\left(\frac{\tau}{\tau}\right)^{1 / 4}, a=\frac{1-(\tau / \tau}{1+(\tau / \tau}\right)^{1 / 2} \\
\tau_{C}=C / G \text { and } \tau_{L}=L R \text {. }
\end{gathered}
$$

Note that the frequency dependence of $Z_{0}(z)$ is only influenced by the quotient $\tau_{L} / \tau_{c}$. The power series expansion of $Z_{0}(z)$ may be obtained as follows. In a first step, the expression $(1+a z)(1-a z)$ is expanded into its power series. It should be noted that the power series expansion of a rational function $H(z)=p(z) / q(z)$ corresponds to the impulse response of a discrete system with transfer function $H(z)$. Finally, to obtain the power series expansion of $Z_{0}(z)$, the following result may be used.

Given $f(z)=\sum_{i=0}^{\infty} \frac{a_{i}}{i !} z^{i}$, the function $g(z)=f(z)^{\prime \prime 2}$ may be expressed as

$g(z)=\sum_{i=0}^{\infty} \frac{b_{i}}{i !}{ }^{i}$, where $b_{0}=a_{0}{ }^{1 / 2}, b_{1}=0.5 a_{1} / b_{0}, \quad b_{2}=\left(0.5 a_{2}-b_{1}{ }^{2}\right) b_{0}$

$b_{3}=\left(0.5 a_{3}-3 b_{1} b_{2}\right) / b_{0}, \quad b_{4}=\left(0.5 a_{4}-3 b_{2}^{2}-4 b_{1} b_{2}\right) / b_{0}, \quad b_{5}=\left(0.5 a_{5}-10 b_{2} b_{3}-5 b_{1} b_{4}\right) / b_{0}$.

$b_{6}=\left(0.5 a_{6}-10 b_{3}{ }^{2}-15 b_{2} b_{4^{-}}-6 b_{1} b_{5}\right) / b_{0}, b_{7}=\left(0.5 a_{7}-35 b_{3} b_{4}-21 b_{2} b_{5}-7 b_{1} b_{6}\right) / b_{0}, \ldots$

It has been found that the multipoint Padé approximation obtained from the power series of $Z_{0}$ about $z=0$, along with the function value of $Z_{0}$ at $z=1$ and $z=-1$ (corresponding to $f \rightarrow \infty$ and $f=0$ ) gives satisfactory results if the distance between $1 / \tau L$ and $1 / \tau_{C}$ is less than 3 to 4 decades. Better accuracy may be obtained by including some terms of the power series expansion about the points $z=\left(s 0-1 / \tau_{l}\right) /\left(s 0+1 / \tau_{L}\right)$ and $z=\left(s_{0}-1 / \tau_{C}\right) /\left(s_{0}+1 / \tau_{C}\right)$.

After the propagation function $F(s)$ is decomposed in the form $F(s)=F C(s) \exp (-\tau s)$, where $\tau=l(L C)^{\prime \prime 2}$, it is possible to apply the same procedure to the function $F_{d}(s)$. After taking the same bilinear transformation, we obtain

$$
F_{c}(z)=\exp \left[-l \sqrt{R G} \cdot \frac{1}{b(1+z)}\left(\sqrt{1-(a z)^{2}}-b(1-z)\right)\right], \text { where } b=\frac{(\tau / \tau)^{1 /}}{1+(\tau / \tau)}
$$

To obtain the power series expansion of Fc(z), the following additional result is useful.

$$
\begin{aligned}
& \text { Given }, f(z)=\sum_{i=0}^{\infty} a_{i} z^{i} \text { the function } g(z)=\exp (f(z)) \text { may be expressed as } \\
& g(z)=\sum_{i=0}^{\infty} c_{i} z^{i}, \text { where } c_{0}=\exp \left(a_{0}\right) \quad \text { and } c_{n}=\frac{1}{n} \sum_{k=1}^{n} k \cdot c_{n-k} a_{k} .
\end{aligned}
$$

It should be noted that when the power series expansion has to be carried out about $z=z_{0}$, the same procedures may be used introducing a new variable $u=z-z_{0}$ and expanding in powers of $u$. As observed in approximating the characteristic impedance, providing the power series about $z=0$ and the function values for $z=1$ and $z=-1$ gives good approximation over the whole frequency axis while it is possible to increase accuracy including information of other z-plane points. 
Inclusion of skin-effect

A simple and yet accurate skin-effect model [6] in the form of $Z s=K s^{1 / 2}$ may be included in the described method, yielding

$$
Z_{o}(s)=\sqrt{(L s+R+K \sqrt{s}) / C s} \text { and } \theta(s)=l \sqrt{(L s+R+K \sqrt{s}) C s} .
$$

Applying the bilinear transformation to $Z o(s)$ and $\mathrm{Fc}(\mathrm{s})$ with a generic value of $\mathrm{s}_{0}$ gives the expressions

$$
\begin{array}{r}
Z_{o}(z)=\sqrt{\frac{R(1+z)+L s_{0}(1-z)+K \sqrt{s_{0}} \sqrt{1-z^{2}}}{C s_{0}(1-z)}} \\
F_{c}(z)=\exp \left\{-l \frac{1}{1+z}\left[\left[L C s_{0}^{2}\left(1-2 z+z^{2}\right)+K C s_{0}^{\frac{1}{2}}(1-z)\left(1-z^{2}\right)^{\frac{1}{2}}+\right.\right.\right. \\
\left.\left.\left.R C s_{o}\left(1-z^{2}\right)\right]^{\frac{1}{2}}-\sqrt{L C} s_{o}(1-z)\right]\right\}
\end{array}
$$

where repeated application of the above described results allows to obtain the power series expansion required for the multipoint Padé procedure.

\section{Application Examples}

The described method has been applied to different types of transmission lines. Two examples and a comparison with the results obtained using other techniques are given.

\section{Example 1.}

A $10 \mathrm{~cm} R L C G$ transmission line with parameters $\mathrm{R}=2.5 \Omega / \mathrm{cm}$, $\mathrm{L}=10 \mathrm{nH} / \mathrm{cm}, \mathrm{C}=4 \mathrm{pF} / \mathrm{cm}$ and $\mathrm{G}=0.5 \mathrm{mS} / \mathrm{cm}$ has been modeled. This example is described in [1] and [2]. Using the explicit technique in [1], a fourth order rational approximation for the characteristic impedance yields $0.01 \%$ error in magnitude and $0.0 \mathrm{l}^{\mathrm{H}}$ error in phase. The computed approximation errors for the characteristic impedance and propagation function $\mathrm{Fc}(\mathrm{s})$ are shown in figure 5 , where the same order (4) has been maintained. The proposed technique gives only $10 \%$ peak error in magnitude and $10^{\text {in }}$ peak error in phase. Alternatively, for a prescribed accuracy, the order of the approximation may be much lower. While the same degree of approximation may also be obtained making use of optimization algorithms [2], [7], it should be emphasized that the described algorithm is explicit in the sense that no iterative procedure has to be carried out at any stage.

\section{Example 2.}

A $96.8 \mathrm{~m} \mathrm{RG}-21$ coaxial cable with frequency dependent parameters characterized with the skin-effect model $K s^{\prime \prime 2}$ has also been studied. Cable parameters are $Z o=(L / C)^{12}=53 \Omega$, $\mathrm{R}=0.25 \Omega / \mathrm{m}, \mathrm{K}=25.10^{\circ} \Omega \mathrm{s}^{1 / 2} / \mathrm{m}, 1 /(L C)^{1 / 2}=2.10^{\circ} \mathrm{m} / \mathrm{s}$. The same cable is modeled in [3] where, after an accurate initial estimate of the zeros and poles of a second order rational function, a GaussMarquardt optimization technique is used to adjust only the magnitude function of the characteristic impedance function in the frequency range $1 \mathrm{KHz}$ to $1 \mathrm{GHz}$. With the explicit technique described, the magnitude and phase function are simultaneously adjusted (figure 6). As expected, a reduction in the phase error is obtained by allowing the error in magnitude to increase accordingly. Again, the proposed technique yields similar performance while avoiding the difficulties inherent to optimization procedures.

\section{Conclusions}

In this paper, a systematic non-iterative method for obtaining models of lossy and dispersive transmission lines, allowing the inclusion of skin effect parameters modeled as $K s^{\prime 2}$, is described. The technique, based on multipoint Padé approximants, does not require the use of any optimization technique -overcoming problems inherent to optimization algorithms, such as initial parameter estimation or convergence to local minima- and achieves the same approximation error as other existing methods that do require optimization techniques. The obtained model may be implemented directly on any standard circuit simulation program, such as SPICE.
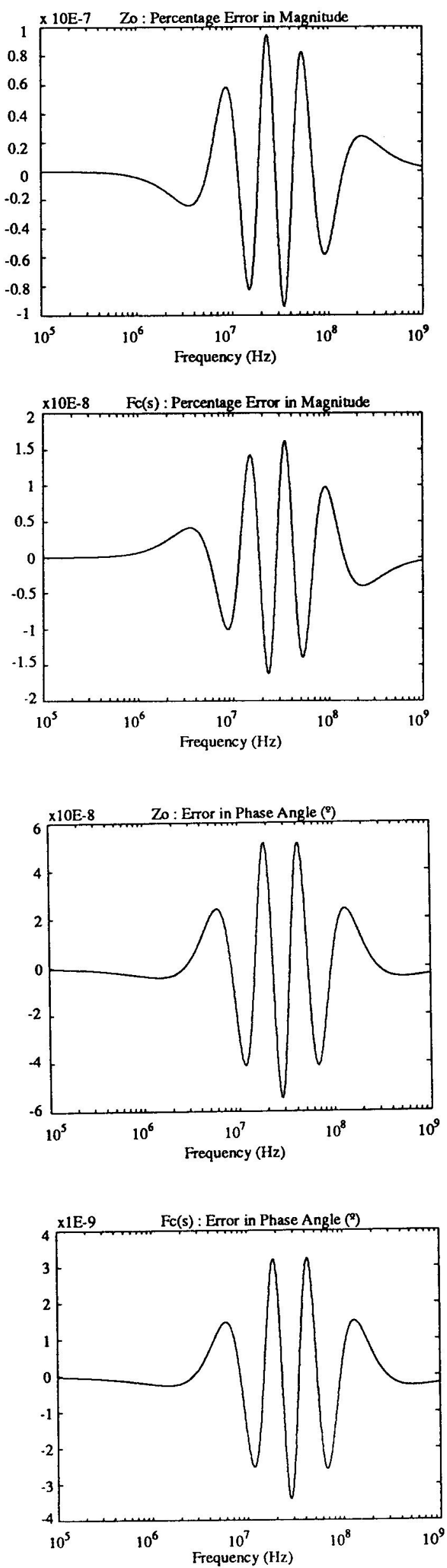

Fig. 5 


\section{References}

[1] F.Y. Chang, "Waveform Relaxation Analysis of RLCC Transmission Lines." Nov. 1990. IEEE Trans. Circuits Syst., vol. 37, pp. 1394-1415.

[2] J. Alonso, J. Borja and F. Perez, "A Universal Model for Lossy and Dispersive Transmission Lines for Time Domain CAD of Circuits." May 1992. IEEE Trans. Microwave Theory Tech., vol. 40, pp. $938-946$.

[3] F.Y. Chang, "Waveform Relaxation Analysis of Nonuniform Lossy Transmission Lines Characterized with FrequencyDependent Parameters." Dec. 1991. IEEE Trans. Circuits Syst., vol. 38, pp. $1484-1500$.

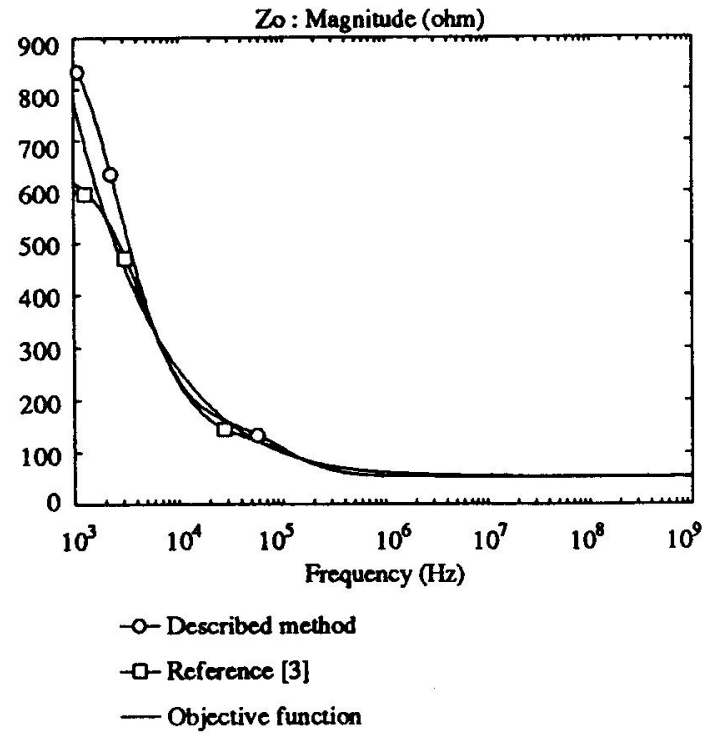

[4] N. Nagai, "Linear Circuits, Systems and Signal Processing." 1990 Marcel Dekker, New York.

5] G.A. Baker, Jr., and P.R. Graves-Morris, "Padé Approximants, Part 1: Basic Theory, Part 11: Extensions and Applications." 1981 Addison Wesley, London.

[6] L.-C. Calvez and J. Le Bihan, "Coefficient Algorithms for Time-Domain Response of Skin Effect Lossy Coaxial Cables with Arbitrary Resistive Termination." Sep. 1986. IEEE Trans. Circuits Syst., vol. 33, pp. 915-920.

[7] J.M. Miró and P. Palà, "CiOpt: a Program for Optimization of the Frequency Response of Linear Circuits." May 1991. Proceedings of the 34th Midwest Symposium on Circuits and Systems, Monterey California, pp. 1039-1042.

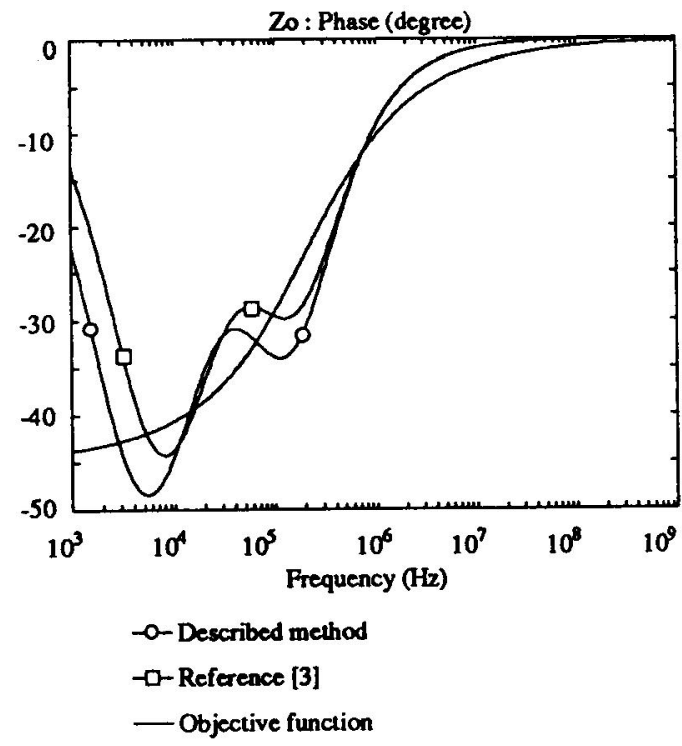

Fig. 6 